Estudios Románicos, Volumen 29, 2020, pp. 93-119

ISSN: 0210-4911

eISSN: 1989-614X

DOI: https://doi.org/10.6018/ER.424571

\title{
LE VOCABULAIRE DE L'ARCHITECTURE \\ DANS LE DICTIONNAIRE DE L'ACADÉMIE \\ FRANÇAISE (1762) : APRÈS LES TRACES DE NICOT
}

(The vocabulary of architecture in the

Dictionary of the French Academy (1762): after Nicot's traces)

\author{
Zaida Bartolomé-Díaz* \\ Universidad de Las Palmas de Gran Canaria \\ Verónica C. Trujillo-González ${ }^{* *}$ \\ Instituto Universitario de Análisis y Aplicaciones Textuales (IATEXT) \\ Universidad de Las Palmas de Gran Canaria
}

\begin{abstract}
Studies on the lexicographical treatment of technical architectural terms in general language dictionaries are scarce. Our article shows how architectural technical terms were treated by the French Academy in the fourth edition of its dictionary (1762) through the study of a series of specific terms related to architecture and building. Jean Nicot's Thresor de la langue françoyse, tant ancienne que moderne (1606) is considered to be one of the first dictionaries to have identified a large number of architectural terms. In this work, we trace the presence or absence of these terms in the Dictionnaire de l'Académie française (1762) and study whether they have become part of the common language or whether they continue to be regarded as specialized terms.
\end{abstract}

Keywords: Dictionnaire de l'Académie (1762) ; Thresor de la langue françoyse (1606) ; Nicot ; architectural terms ; marks ; domains ; common language ; technical language.

Résumé : Les études sur le traitement lexicographique des termes techniques d'architecture dans les dictionnaires de langue générale sont rares. Notre article montre comment les termes techniques concernant l'architecture ont été traités par l'Académie française dans la quatrième édition de son dictionnaire (1762) à travers l'étude d'une

*Adresse pour la correspondance : Zaida Bartolomé Díaz. Universidad de Las Palmas de Gran Canaria. Campus del Obelisco. Pza. de la Constitución, s/n. 35003 Las Palmas de Gran Canaria (zaida.bartolome101@alu.ulpgc.es).

** Adresse pour la correspondance : Verónica Trujillo González. Instituto Universitario de Análisis. Instituto Universitario de Análisis y Aplicaciones Textuales (IATEXT). Universidad de Las Palmas de Gran Canaria. Campus del Obelisco. Pza. de la Constitución, s/n. 35003 Las Palmas de Gran Canaria (veronica.trujillo@ulpgc.es). 
série de termes spécifiques liés à l'architecture et au bâtiment. Le Thresor de la langue françoyse, tant ancienne que moderne de Jean Nicot (1606) est considéré comme l'un des premiers dictionnaires à avoir identifié un grand nombre de termes architecturaux. Dans cet ouvrage, nous retraçons la présence ou l'absence de ces termes dans le Dictionnaire de l'Académie française (1762) et étudions s'ils sont devenus partie intégrante de la langue commune ou s'ils continuent à être considérés comme des termes spécialisés.

Mots clés : Dictionnaire de l'Académie (1762); Thresor de la langue françoyse (1606); Nicot ; termes d'architecture ; marques ; domaines ; langue commune ; langue technique.

\section{Introduction}

De nombreux travaux s'intéressent au lexique commun dans les dictionnaires scientifiques et techniques : Josselin (2005), Phal (1969), Cordero Monge (2009), Rodríguez Adrados (1997), Boulanger et L'Home (1991), Courbières et Fraysse (2009).

De même, il existe un nombre important de travaux consacrés à la présence des termes de spécialité dans les dictionnaires généraux : Eluerd (1999), Rouleau (2003), Ridao Rodrigo et al. (2016), Terral (2004), Tetet (1994); cependant, ces travaux font référence, dans la plupart des cas, à des termes de domaines tels que l'informatique, la médecine ou le droit. Ainsi, il existe, à notre connaissance, un manque important de travaux concernant la présence du vocabulaire propre au domaine du bâtiment et de l'architecture dans les dictionnaires généraux de la langue française. Le travail de Wooldrige (1985) est, cependant, une exception. En effet, cet auteur étudie le dictionnaire de Jean Nicot, le Thresor de la langue françoyse, tant ancienne que moderne (1606), dorénavant le D.N, pour retracer l'évolution du vocabulaire de l'architecture dans la série Estienne - Nicot ${ }^{1}$ à travers les citations des sources, notamment de Vitruve ${ }^{2}$. Comme l'explique Wooldridge (1985), les ouvrages lexicographiques de la Renaissance citent abondamment l'œuvre de Vitruve en contribuant à introduire certains termes propres à l'architecture dans la langue courante.

Dans son étude, Wooldrige (1985) signale que les marques d'usage utilisées dans le dictionnaire de Nicot (1606) constituent une approche des différents vocabulaires thématiques. Ainsi, pour l'extraction terminologique qu'il réalise dans ce dictionnaire, il se concentre sur les marques d'usage liées au vocabulaire des métiers de la construction et de l'art de construire: «terme de maçon », «mot usité en maçonnerie », « en fait de maçons », « les maçons en usent par metaphore », " en massonnerie », " ainsi par les massons dit », « en matiere de menuiserie », « en cas de menuyserie », « en fait de charpenterie », " mot fort usité entre charpentiers », « les charpentiers par metaphore en usent », « que les architectes nomment » et « en cas d'architecture ».

\footnotetext{
1 Le dictionnaire de Nicot (1606) est, d'après Wooldrige (1985) la cinquième édition du Dictionaire françois-latin de Robert Estienne (1539).

2 Architecte et ingénieur du premier siècle avant J.-C., Vitruve rédige le traité De architectura, traduit en français par Jean Martin en 1547.
} 
De cette manière, Wooldrige (1985), à travers l'analyse de la présence simultanée de deux ou de plusieurs de ces marques dans le même article, recense une liste de 94 termes qui véhiculent l'essentiel du vocabulaire du bâtiment dans le répertoire de Nicot (1606). Il faut préciser, nonobstant, que pour établir cette liste, Wooldridge (1985) prend en compte le texte complet de Nicot et non pas seulement les entrées du dictionnaire, car, comme explique Bloch (1904), une bonne partie du vocabulaire français du Thresor n'est pas traitée dans sa nomenclature.

L'extraction terminologique réalisée par Wooldridge (1985) nous sert donc de point de départ de notre étude. Effectivement, nous partons de l'hypothèse que ces 94 termes identifiés par Wooldrige constituent le premier constat d'une introduction massive des termes spécialisés liés au domaine de l'architecture dans un dictionnaire général de langue. Notre étude vise à savoir si ces 94 termes sont répertoriés dans la 4ème édition du Dictionnaire de l'Académie Française (1762) et s'ils sont devenus des mots de la langue courante ou continuent à être considérés comme des termes spécialisés propres au domaine de l'architecture et du bâtiment. De même, nous allons analyser le traitement lexicographique que ces termes ont reçu dans le répertoire de l'Académie. Le choix de la 4ème édition du dictionnaire institutionnel se justifie car ce dictionnaire répertorie, pour la première fois, un grand nombre des mots techniques, suite aux grands changements techniques et scientifiques de l'époque. Pour notre étude nous avons donc choisi deux époques charnières de l'histoire de la lexicographie, la première correspond à l'introduction massive de termes propres à l'architecture dans un dictionnaire général de langue au XVII e siècle, le dictionnaire de Nicot et la deuxième, un siècle et demi plus tard, lorsque l'Académie modifie son critère de recensement de mots et décide d'introduire un grand nombre de mots techniques dans la $4^{\text {ème }}$ édition du répertoire institutionnel.

En outre, afin de confirmer l'aspect synchronique des mots répertoriés comme propres au domaine de l'architecture et d'évaluer les définitions fournies par l'Académie, nous allons utiliser deux autres dictionnaires contemporains du Dictionnaire de l'Académie (1762) : le Dictionnaire portatif des beaux-arts de Jacques Lacombe (1752) qui, selon Roffidal (2012) est un ouvrage technique et représentatif de la langue de son époque, et le Dictionnaire d'architecture civile, militaire et moderne et de tous les arts et métiers qui en dépendent, de Roland le Virloys, (1770) qui, d'après nos recherches, est le seul ouvrage de ce siècle consacré entièrement à l'architecture.

De même, nous partons de l'hypothèse que ces termes sont répertoriés dans le Dictionnaire de l'Académie française (1762) avec un marquage diatechnique spécifique, c'est-à-dire qu'ils sont considérés comme représentatifs des métiers de la construction et de l'art de construire. Cependant, il est aussi possible que certains de ces termes spécialisés ne contiennent pas de marquage car le système de marquage à cette époque n'est pas encore complètement établi. Notre analyse vise donc à déterminer si certains de ces termes sont devenus des mots de la langue courante ou s'ils sont considérés, d'après l'étude de leur définition comme des mots techniques. 


\section{La lexicographie en France au XVIII ${ }^{e}$ siècle et la quatrième édition du dictionnaire de l'Académie (1762)}

Le XVIII ${ }^{\mathrm{e}}$ siècle est une période fondamentale pour l'histoire de la lexicographie en France. Effectivement, deux faits marquent indéniablement cette période de l'histoire : d'une part, la publication de l'Encyclopédie, à partir de 1751, dont l'influence sur les ouvrages de l'époque et postérieurs est incontestable et, d'autre part, la forte production lexicographique présente tout au long du siècle des Lumières qui témoigne de l'évolution des idées et des progrès techniques du moment.

Quant à l'Académie, comme le signale Trujillo-González (2019), le XVIII' siècle est la période la plus riche et variée de toute son histoire, car quatre nouvelles éditions du Dictionnaire de l'Académie (1718, 1740, 1762 et 1798) et un supplément à l'édition de $1798^{3}$ voient le jour, ce qui restera un geste inédit jusqu'à nos jours.

En ce qui concerne l'édition de 1762, il faut souligner qu'il s'agit de l'édition dans laquelle, pour la première fois, l'Académie décide de répertorier les mots techniques afin d'en refléter les usages de l'époque. Pour Quemada (1985: 79) cette édition est particulièrement importante :

L'édition de 1762 est encore plus importante. (...). L'Académie, par ailleurs soucieuse de refléter une certaine image des transformations de l'usage réel (il avait beaucoup changé au cours du dernier demi-siècle), ouvre ses colonnes aux vocabulaires des sciences, des arts et des métiers. Ce faisant, elle témoigne, à sa façon, des conséquences linguistiques du grand brassage des hommes et des idées et de l'expansion des sciences et des techniques dans la norme d'usage des nouvelles classes dominantes.

Effectivement, l'édition de 1762 marque un point d'inflexion dans les principes qui guident la sélection du matériel lexique pour le dictionnaire des Immortels, ce qui se reflète dans la préface que Duclos a rédigée pour cette édition ${ }^{4}$ :

Les sciences et les arts ayant été plus cultivés et plus répandus depuis un siècle qu'ils ne l'étoient auparavant, il est ordinaire d'écrire en François sur ces matières. En conséquence plusieurs termes qui leur sont propres, et qui n'étoient autrefois connus que d'un Petit nombre de personnes, ont passé dans la Langue commune. Auroit-il été raisonnable de refuser place dans notre Dictionnaire à des mots qui sont aujourd'hui d'un usage presque général? Nous avons cru devoir admettre dans cette nouvelle édition, les termes élémentaires des sciences, des arts et même ceux des métiers, qu'un homme de lettres est dans le cas de trouver dans des ouvrages où l'on ne traite pas expressément des matières auxquelles ces termes appartiennent.

3 La finalité de ce supplément est de mettre à jour le vocabulaire avec les changements des signifiés des mots ainsi que d'ajouter les néologismes issus de la période révolutionnaire.

$4 \quad$ On cite à travers Eluerd (1999). 
Cet extrait de la préface met en lumière la position adoptée par les Immortels face aux mots issus des sciences, des arts et des métiers que l'on trouve dans les ouvrages non spécialisés. Ce changement d'avis en faveur de l'introduction des termes techniques dans le dictionnaire général de langue est accompagné de la nécessité d'ouverture de l'Institution à des savants d'autres domaines, notamment, à des membres de l'Académie Royale des Sciences, entre autres ${ }^{5}$, car il ne suffit pas d'intégrer ces nouveaux mots dans la nomenclature du répertoire institutionnel, il reste impératif de les définir. Ainsi, ces nouveaux élus inciteront l'Académie à introduire, dans la quatrième édition (1762), des milliers de mots appartenant à des domaines spécialisés. Pour Eluerd (1999 : 66) ce qui est réellement remarquable dans cette période est l'intégration de mots techniques dans le discours général des dictionnaires :

Ce qui est vraiment nouveau au XVIIIe siècle, c'est moins le développement considérable des vocabulaires scientifiques et techniques que leur pénétration dans leurs discours commun. Et s'il y a rapport de cause à effet, ce n'est pas une simple question de quantité de mots, une sorte de débordement d'un trop-plein, mais une disposition nouvelle entre les usages scientifiques et techniques du langage et l'usage ordinaire. (...). Cette nouvelle disposition ne se lit pas que dans la présence ou le dénombrement des vocabulaires spécialisés, il faut aussi interroger leurs emplois et leur réception.

L'édition de 1762 est, par conséquent, une édition incontournable si l'on veut étudier le passage des termes techniques à la langue courante. Elle s'avère un témoin privilégié des changements sociaux et culturels vécus au siècle des Lumières.

\section{Le problème du manque de systématisation des informations dans les définitions lexicographiques au XVIII ${ }^{e}$ siècle}

Une des difficultés liées à l'étude des dictionnaires anciens repose sur le manque de systématisation des informations. Même si la situation s'améliore au XVIII ${ }^{e}$ siècle, car il s'agit précisément de la période où la standardisation de la langue commence à prendre forme, c'est un des éléments qu'il faut encore considérer lors des analyses des répertoires. Par conséquent, l'importante pénétration des termes de spécialités dans les ouvrages du XVIII siècle n'est pas facilement repérable. En effet, comme le remarque Wionnet (1998: 334), les méthodes lexicographiques du XVIII ${ }^{e}$ siècle ne sont pas exposées systématiquement. Ainsi, les préfaces et les libellés qui accompagnent la parution des dictionnaires comportent uniquement des indications sur le genre de l'ouvrage et sur les objectifs visés. Les critères suivis pour l'inclusion des technolectes et le traitement que ces mots ont reçu restent, de cette façon, dans l'ombre.

\footnotetext{
5 Pour connaître mieux ces lexicographes issus d'autres disciplines que la littéraire, $c f$. Eluerd (1999).
} 
De surcroît, l'information contenue dans les définitions et la manière dont elle s'organise nécessite un savoir-faire lexicographique qui est encore en construction. En effet, souvent les critères définissant le marquage de spécialité ne sont pas clairement établis ni exposés nulle part.

Cela peut s'expliquer par plusieurs raisons ${ }^{6}$ :

I. Il y a une omission involontaire du marquage.

II. L'appartenance du terme à plusieurs domaines d'activité force le lexicographe à en choisir un au détriment des autres.

III. La difficulté à déterminer s'il s'agit d'un terme de spécialité ou d'un mot de la langue courante.

Pour notre étude, les marquages diatechniques ou marques de spécialité sont fondamentales. Cependant, dans la 4ème édition du Dictionnaire de l'Académie (1762), ces marques de spécialité, bien qu'existantes, ne sont ni définies ni expliquées dans la préface. Ces problèmes liés au marquage renforcent donc le besoin d'étudier et de retracer l'origine et l'évolution de ces termes de spécialité. La liste des termes établie par Wooldridge (1985) contenant l'essentiel du vocabulaire relatif au domaine du bâtiment nous permettra, en outre de vérifier la présence ou l'absence de ces termes dans le répertoire institutionnel, d'étudier le type de marquage employé.

\section{Description du corpus et méthodologie}

Pour mener à bien notre travail, nous avons constitué un corpus d'étude formé par les 94 termes qui, d'après Wooldridge (1985), véhiculent l'essentiel du vocabulaire du bâtiment dans le Thresor de la langue françoyse, tant ancienne que moderne, de Jean Nicot, dorénavant D. N. (1606).

Ces 94 termes, listés ci-dessous tels qu'ils apparaissent dans le D.N., c'est-à-dire avec leur graphie originale, représentent l'essentiel du vocabulaire du bâtiment présent dans le répertoire de Nicot (1606) :

6 Nous utilisons et adaptons au contexte particulier de notre étude les raisons établies par Turcan et Wooldridge (2002). 
Le vocabulaire de l'architecture dans le Dictionnaire de l'Académie française (1762) :

après les traces de Nicot

\section{Tableau 1.94 termes du Thresor contenant l'essentiel du vocabulaire du bâtiment (Wooldridge, 1985)}

\begin{tabular}{|c|c|c|c|}
\hline acroteres & entablement & 65. & paroy \\
\hline adents & espauletées & 66. & parpaigne \\
\hline ais & estage & 67. & pavé \\
\hline appuy & estalon & 68. & pivot \\
\hline architrave & fenestrage & 69. & place \\
\hline assemblage & fenestre & 70. & planche \\
\hline assembler & frit & 71. & plancher \\
\hline assiette & frize & 72. & plane \\
\hline astragale & gâche & 73. & plant \\
\hline auge & gargouille & 74. & plateforme \\
\hline augee & gascher & 75. & plinthe \\
\hline bâcler & herce & 76. & plomb \\
\hline baffroy & 45. & 77. & plommer \\
\hline barriere & hire & 78. & portail \\
\hline bâtant & huis & 79. & porte \\
\hline bourdonniere & jambe & 80. & poultre \\
\hline brique & larmier & 81. & pourpris \\
\hline chaas & late & 82. & queue d'aronde \\
\hline chardoniere & later & 83. & rez \\
\hline charpentier & ligne & 84. & sapper \\
\hline chasteau & mardélle & 85. & severonde \\
\hline clou & marqueter & 86. & sole \\
\hline comble & marrein & 87. & solive \\
\hline court & masson & 88. & sommier \\
\hline crapaudine & menuiserie & 89. & tariere \\
\hline créneaux & menuisier & 90. & tourillon \\
\hline crespir & moise & 91. & tourrion \\
\hline croisée & moitoyen & 92. & travée \\
\hline dosserasse & montant & 93. & tuile \\
\hline embraser & mortier & 94. & villebrequin \\
\hline embrasure & muel & & \\
\hline enduict & niveau & & \\
\hline
\end{tabular}

Nous avons réalisé pour chacun de ces termes une recherche simple dans la version électronique de la 4ème édition du Dictionnaire de l'Académie $(1762)^{7}$, dorénavant D.A.,

$7 \quad$ Nous travaillons avec l'édition électronique du dictionnaire qui permet de réaliser une recherche avancée et d'obtenir tous les mots appartenant au domaine de l'architecture : https://academie.atilf.fr/4/ [dernière date de consultation : 15-03-20]. 
afin de vérifier s'ils font partie du dictionnaire institutionnel et quel traitement lexicographique ils y ont reçu.

Nous avons confirmé, également, la présence ou l'absence de chacun de ces termes dans les deux dictionnaires spécialisés contemporains du D.A., c'est-à-dire, le Dictionnaire portatif des beaux-arts (1752) et le Dictionnaire d'architecture civile, militaire et moderne et de tous les arts et métiers qui en dépendent (1770), afin de déterminer s'ils sont encore en usage et s'ils étaient utilisés ou pas dans des domaines spécialisés.

La méthodologie adoptée pour cette étude s'encadre ainsi dans une approche métalexicographique mixte quantitative et qualitative. Nous avons structuré notre analyse en trois étapes :

- lère étape : étude de la présence des termes du corpus dans le Dictionnaire de l'Académie (1762).

- 2ème étape : étude quantitative et lexicographique des termes du corpus présents dans le Dictionnaire de l'Académie (1762).

- 3ème étape : étude comparative entre les définitions de l'Académie et les deux autres dictionnaires techniques des articles qui portent un marquage diatechnique indiquant son appartenance aux domaines du bâtiment et de l'architecture.

\section{5. Étude de la présence des termes du corpus dans le Dictionnaire de l'Académie (1762)}

Dans cette première étape, nous voulons déterminer la présence ou l'absence dans le D.A. des 94 termes qui composent notre corpus. Pour cela, il est nécessaire de réaliser une vérification des possibles modifications orthographiques des termes absents, afin de préciser quels mots figurent dans le D.A., mais avec une graphie modifiée. Effectivement, comme l'indique Farid (2012), au fil des réformes successives et depuis sa création, l'Académie française essaie d'établir des règles pour l'orthographe française, motivées par l'apparition d'une " nouvelle orthographe " simplifiée, nécessaire pour l'utilisation des imprimeurs qui amène, comme par exemple dans le Dictionnaire de Richelet en 1680, à simplifier des consonnes doubles et à supprimer des lettres qui avaient été rajoutées y compris des lettres grecques.

Pour Giovani (2006), déjà dans la troisième édition du D.A. en 1740, le nombre de lettres étymologiques est considérablement réduit, un emploi conséquent des accents est envisagé et l'usage de la lettre « $y$ » est réglementé, de sorte que dans cette édition la graphie de 6177 mots change.

D'après Sallenave, (2016) :

Au XVIII ${ }^{e}$ siècle, de grands changements ont lieu à partir de 1740, quand les philosophes entrent à l'Académie. Plus du quart du vocabulaire est transformé et modernisé, par suppression de lettres inutiles (h : autheur > auteur, authorité > autorité), des consonnes muettes (adjouster $>$ ajouter, adveu $>$ aveu, debvoir $>$ devoi r), malgré quelques oublis (sculpteur, baptême), remplacement du es interne marquant 
Le vocabulaire de l'architecture dans le Dictionnaire de l'Académie française (1762) : après les traces de Nicot

la prononciation par ê (estre > être) ; en 1762 (seulement !), mise en place de l'accent grave. Voltaire fait adopter l'orthographe ai au lieu de oi (françois, anglois), fait corriger les formes verbales j'estois, je feroi, je finirois, etc.

Ainsi, en prenant en compte ces évolutions de l'orthographie française, nous concluons que neuf termes de notre corpus ne figurent pas dans la 4ème édition du D.A. : adents, bourdonniere, chardoniere, espauletées, hire, marrein, muel, severonde, tourrion.

Afin de vérifier, si à l'époque, ces termes étaient encore en usage dans le domaine de l'architecture et du bâtiment, nous avons consulté le Dictionnaire portatif des beaux-arts de Jacques Lacombe (1752) et le Dictionnaire d'architecture civile, militaire et moderne et de tous les arts et métiers qui en dépendent de Roland le Virloys (1770). Des neuf termes consultés, il n'y en a qu'un qui soit répertorié : Hire. Cependant, ce terme se trouve dans le Dictionnaire d'architecture civil dans l'article qui fait référence au nom de famille de Laurent de la Hire, ce qui implique qu'on ne peut plus le considérer comme un terme propre au domaine de l'architecture. Ainsi, nous pouvons confirmer que ces neuf termes ne sont plus d'usage dans la deuxième moitié du XVIII ${ }^{\mathrm{e}}$ siècle.

En ce qui concerne les modifications orthographiques, nous avons constaté la présence de 33 termes de notre corpus enregistrés dans le D.A. mais avec une graphie modifiée :

Tableau 2. 33 termes de notre corpus figurèrent dans la 4ème édition du D.A. avec une graphie modifiée.

\begin{tabular}{|l|l|l|}
\hline 1. Acroteres $->$ Acrotères & 2. Appuy $->$ Appui & 3. Augee $->$ Augée \\
\hline 4. Baffroy $->$ Beffroi & 5. Barriere $->$ Barrière & 6. Battant $->$ Bâtant \\
\hline 7. Chaas $->$ Chas & 8. Chasteau $->$ Château & 9. Court $->$ Cour \\
\hline 10. Créneaux $->$ Créneau & 11. Crespir $->$ Crépir & 12. Dosserasse $->$ Dosseret \\
\hline 13. Enduict $->$ Enduit & 14. Estage $->$ Étage & 15. Estalon $->$ Étalon \\
\hline 16. Fenestrages $->$ Fenêtrage & 17. Fenestre $->$ Fenêtre & 18. Frize $->$ Frise \\
\hline 19. Gascher $>$ Gâcher & 20. Herce $->$ Herse & 21. Late $->$ Latte \\
\hline 22. Latter $->$ Later & 23. Mardélle $->$ Margelle & 24. Masson $->$ Maçon \\
\hline 25. Moitoyen $->$ Mitoyen & 26. Paroy $->$ Paroi & 27. Parpaigne $->$ Parpaing \\
\hline 28. Plateforme $->$ Plate-forme & 29. Plommer $->$ Plomber & 30. Poultre $->$ Poutre \\
\hline 31. Sapper $->$ Saper & 32. Tariere $->$ Tarière & 33.Villebrequin- $>$ Vilebrequin \\
\hline
\end{tabular}

Pour le cas du mot Mardélle, notons que le D.A. recueille la graphie Mardelle, sans l'accent, mais il ne la définit pas, il nous renvoie directement au mot Margelle (Voyez MARGELLE).

Le tableau suivant montre le pourcentage des termes présents et absents de notre corpus dans le D.A. : 
Tableau 3. Présence des Termes du corpus dans le D.A.

\begin{tabular}{|c|c|c|}
\hline $\begin{array}{c}\text { Total de termes } \\
\text { du corpus }\end{array}$ & $\begin{array}{c}\text { Termes du corpus } \\
\text { présents dans le D.A. }\end{array}$ & $\begin{array}{c}\text { Termes du corpus } \\
\text { absents du D.A. }\end{array}$ \\
\hline $\begin{array}{c}94 \\
(100 \%)\end{array}$ & 85 & 9 \\
$(90,5 \%)$ & $(9,5 \%)$ \\
\hline
\end{tabular}

Ainsi, en ce qui concerne la présence des 94 mots de notre corpus dans le D. A., il y a 9 mots, soit $9,5 \%$ des termes qui ne sont pas présents dans la 4ème édition du D.A. et 85 termes, soit $90,5 \%$, qui sont répertoriés dans le dictionnaire institutionnel.

Parmi les 85 mots qui font partie de la nomenclature du répertoire institutionnel, nous avons constaté que 52 de ces termes, soit $61 \%$, n'ont subi aucune modification orthographique tandis que 33 mots, $38 \%$, figurent avec une graphie modifiée.

\section{6. Étude quantitative et lexicographique des termes du corpus présents dans le Dictionnaire de l'Académie (1762)}

Une fois déterminé les mots de notre corpus présents dans le D.A., 85 mots $^{8}$, nous allons les regrouper par traitement lexicographique et calculer la représentativité de chaque groupe de mots dans l'ensemble du corpus.

En ce qui concerne le marquage diatechnique des termes, il faut remarquer que, même si l'Académie n'explicite pas dans sa préface qu'elle suit un système de marquage précis, après notre analyse, nous pouvons affirmer que dans le répertoire institutionnel il existe bien un système de marquage. Ainsi, nous avons observé que pour marquer les mots concernant un domaine spécialisé, le D.A. utilise le marquage « TERME DE..... ». Voyons un exemple :

DOSSERET. s. m. Terme d'architecture. Petit pilastre saillant.

Tel comme on peut le voir, la définition se limite à une description concise de l'objet sans proposer d'exemples d'utilisation, ce qui constitue en elle-même un type de marque précise. Effectivement, à la différence d'autres entrées du dictionnaire où l'on trouve plusieurs acceptions et exemples, les termes dont le marquage est « TERME DE...» comportent une définition univoque, concise et claire, n'apportant ni exemples ni sens figurés.

Une autre manière de marquer les termes de spécialité utilisée par le D.A. est travers de la marque «EN TERMES DE... ». Dans les articles qui utilisent ce type de marquage on va trouver deux définitions, l'une pour l'usage général, l'autre pour l'usage encore spécialisé, ce qui dénote une certaine vulgarisation du terme. Il n'y a que deux termes d'architecture marqués de cette manière : assembler et assemblage.

8 Pour réaliser cette partie de notre étude, nous avons tenu compte des mots tels qu'ils apparaissent dans le D.A., c'est-à-dire avec leur graphie modifiée si c'est le cas. 
Le vocabulaire de l'architecture dans le Dictionnaire de l'Académie française (1762) : après les traces de Nicot

ASSEMBLAGE. s. m. Amas \& union de plusieurs choses qu'on joint ensemble. Un bateau se fait de l'assemblage de plusieurs pièces de bois. *En termes de Menuiserie, se dit De la manière d'assembler le bois de menuiserie, \& des pièces principales qui servent à cet effet. L'assemblage de cette porte ne vaut rien. Bois d'assemblage. Porte d'assemblage. Il se dit aussi des choses morales. Son caractère est un assemblage de bonnes \& de mauvaises qualités. (sic).

L'exemple utilisé ci-dessus, montre que le style définitoire de la première acception se correspond à celui d'une entrée d'un mot de la langue courante : il n'y a pas de marque diatechnique et on propose un exemple d'utilisation. Par contre, le style définitoire de la deuxième acception ne se correspond pas non plus à celui des termes qui utilisent le marquage «TERME DE.....». En effet, bien que la définition commence par la marque "EN TERMES DE... », elle contient des exemples d'utilisation et propose une utilisation figurée du terme. Il faut également souligner l'utilisation à l'intérieur de l'article de la marque $\left[{ }^{*}\right]$ pour signaler la deuxième acception du terme.

À notre avis, ce type de marque " EN TERMES DE... » est utilisée pour dénoter que soit le terme est en transition depuis un langage spécialisé vers la langue courante, soit, au contraire, il s'agit d'un terme du langage général qui est utilisé par les spécialistes.

Ainsi, on peut signaler, grâce aux marques diatechniques, deux groupes de mots en fonction de leur appartenance au domaine de l'architecture ou du bâtiment, ainsi qu'à d'autres domaines :

Tableau 4. Mots qui portent le marquage diatechnique «TERME DE » ou « EN TERMES DE... »

\begin{tabular}{|c|c|}
\hline $\begin{array}{c}6 \text { termes appartenant aux domaines } \\
\text { du bâtiment et l'architecture }\end{array}$ & $\begin{array}{c}4 \text { termes } \\
\text { appartenant à d'autres domaines }\end{array}$ \\
\hline $\begin{array}{l}\text { dosseret, larmier : } \\
\text { TERME D'ARCHITECTURE }\end{array}$ & $\begin{array}{l}\text { crapaudine: } \\
\text { TERME DE CUISINE }\end{array}$ \\
\hline $\begin{array}{l}\text { moise : } \\
\text { TERME DE CHARPENTERIE }\end{array}$ & $\begin{array}{l}\text { fenêtre: } \\
\text { TERME D'ANATOMIE }\end{array}$ \\
\hline $\begin{array}{l}\text { queue d'aronde: } \\
\text { TERME DE MENUISERIE }\end{array}$ & $\begin{array}{l}\text { montant : } \\
\text { TERME DE FAUCONNERIE }\end{array}$ \\
\hline $\begin{array}{l}\text { assemblage, assembler: } \\
\text { EN TERMES DE MENUISERIE }\end{array}$ & $\begin{array}{l}\text { tourillon: } \\
\text { TERME DE PLUSIEURS ARTS }\end{array}$ \\
\hline Total de termes : $6(7 \%)$ & Total de termes : $4(5 \%)$ \\
\hline
\end{tabular}

Le tableau précédent montre que, des 85 termes recensés dans le D.A., seulement 6 , soit 7\%, sont classés comme appartenant au domaine de l'architecture et du bâtiment. De plus, 4 autres termes, soit $5 \%$, portent un marquage diatechnique propre aux domaines si différents comme l'anatomie, la cuisine ou la fauconnerie. Il faut remarquer cependant, que certains mots, comme par exemple, fenêtre, continuent à faire partie du vocabulaire de l'architecture, même s'ils ne sont pas marqués comme des termes spécialisés dans ce domaine, en raison de leur très large généralisation. 
En ce qui concerne les définitions d'autres termes qui ne portent pas de marquage " TERME DE... » ou « EN TERMES DE...», on remarque que 21 termes suivent le même modèle définitoire de ceux qui la portent. C'est-à-dire que certains mots ont une seule et unique acception liée à l'architecture ou au bâtiment, ce qui, de manière claire, les définit comme appartenant à un domaine spécialisé, sans que pour autant l'Académie leur attribue le marquage «TERME DE...» ou « EN TERMES DE...». À notre avis, ces cas illustrent le manque de stabilité du système de marquage employé par l'Académie, car le type de définition démontre qu'il s'agit encore de mots spécialisés.

Le tableau suivant recense ces mots qui ont une seule acception, univoque, concise et claire, mais qui ne possèdent aucune des marquages diatechniques «TERME DE...» ou « EN TERMES DE...» :

Tableau 5. Mots sans marquage « TERME DE » ou « EN TERMES DE... » mais avec même style définitoire

\begin{tabular}{|c|l|l|}
\hline $\begin{array}{c}\text { Total de termes } \\
\text { du corpus figurant } \\
\text { dans le D.A. }\end{array}$ & $\begin{array}{l}\text { Mots définis avec une définition uni- } \\
\text { voque, concise et claire, n’apportant } \\
\text { ni exemples ni sens figurés }\end{array}$ & $\begin{array}{l}\text { Mots définis avec une définition univoque, } \\
\text { concise et claire, accompagnée d'exemples }\end{array}$ \\
\hline \multirow{2}{*}{85} & $\begin{array}{l}3 \text { mots : acrotères, architrave, par- } \\
\text { paing }\end{array}$ & $\begin{array}{l}18 \text { mots : auge, augée, brique, crépir, char- } \\
\text { pentier, créneau, enduit, gâcher, gargouille, } \\
\text { latte, latter, menuisier, margelle, pourpris, } \\
\text { solive, tarière, travée, vilebrequin }\end{array}$ \\
\cline { 2 - 3 } & $3(3,5 \%)$ & $18(21 \%)$ \\
\hline
\end{tabular}

Nous trouvons ainsi, d'une part, trois mots qui suivent ce style définitoire : définition univoque, concise et claire, n'apportant ni exemples ni sens figurés, sans pour autant porter le marquage «TERME DE...» :

ACROTÈRES. s.m.p. Ce sont des espèces de piédestaux que l'on met d'espace en espace dans les balustrades, de manière que les balustres répondent sur le vuide, \& les acrotères sur le plein.

ARCHITRAVE. s.f. Membre d'Architecture, qui pose immédiatement sur le chapiteau des colonnes ou des pilastres, $\&$ au-dessus duquel est la frise.

PARPAING. s.m. Pierre, moellon qui tient toute l'épaisseur d'un mur, \& dont on voit une face de chaque côté du mur.

Et d'autre part, nous trouvons également 18 autres mots définis avec une seule acception, également univoque, concise et claire, mais accompagnée d'exemples. Voyons un exemple :

BRIQUE. s.f. Terre argilleuse \& rougeâtre, pétrie \& moulée, puis séchée au soleil ou cuite au feu, \& dont on se sert pour bâtir. Carreau de brique. Bâtir de brique. Maison de brique. Bastion revêtu de brique. En Orient, on faisoit cuire la brique au soleil. 
D'après nous, ces 18 entrées devraient également porter la marque diatechnique «TERME DE... » puisqu'elles continuent à être des mots spécialisés, liés à l'architecture dans toutes leurs acceptions.

Ainsi, nous confirmons que, des 85 termes de notre corpus présents dans le D.A., il y en a 10 marqués comme des termes spécialisés, soit $11,75 \%$. De même, on en a identifié 21 sans marquage diatechnique, mais dont les définitions suivent le même modèle définitoire des termes spécialisés portant des marques. Les autres 54 termes, soit 63,5\% des termes étudiés, ne portent aucun marquage diatechnique ni ne suivent non plus le style définitoire des entrées portant ce marquage; cela suggèrerait qu'il ne s'agit plus de termes de spécialité, mais de la langue courante.

Afin de vérifier si cette hypothèse est correcte, nous avons regroupé ces 54 termes selon leurs définitions liées ou pas à l'architecture et au bâtiment:

Tableau 6. Mots qui ne suivent pas le modèle définitoire des entrées portant le marquage « TERME DE » ou « EN TERMES DE... »

\begin{tabular}{|c|c|c|c|c|}
\hline $\begin{array}{c}\text { Total de } \\
\text { termes } \\
\text { du corpus } \\
\text { figurant } \\
\text { dans le } \\
\text { D.A. }\end{array}$ & \begin{tabular}{|c|} 
Mots définis avec \\
plusieurs acceptions, \\
toutes liées aux \\
domaines du bâtiment \\
et de l'architecture \\
et accompagnées \\
d'exemples \\
\end{tabular} & $\begin{array}{l}\text { Mots avec plusieurs accep- } \\
\text { tions dont au moins une } \\
\text { n'est pas liée aux domaines } \\
\text { du bâtiment et de l'archi- } \\
\text { tecture, et accompagnées } \\
\text { d'exemples }\end{array}$ & $\begin{array}{c}\text { Mots avec une } \\
\text { ou plusieurs } \\
\text { acceptions dont } \\
\text { aucune est liée } \\
\text { aux domaines du } \\
\text { bâtiment et de } \\
\text { l'architecture } \\
\end{array}$ & \begin{tabular}{|c|} 
Mots dont les \\
définitions nous \\
indiquent qu'ils \\
sont obsolètes ou \\
bien qui ont été \\
remplacés par \\
d'autres termes \\
\end{tabular} \\
\hline & $\begin{array}{l}7 \text { mots : croisée, } \\
\text { gâche, entablement, } \\
\text { menuiserie, parpaing, } \\
\text { plate-forme, plinthe, } \\
\text { portail }\end{array}$ & $\begin{array}{l}35 \text { mots : ais, astragale, } \\
\text { appui, assiette, bâcler, } \\
\text { barrière, battant, beffroi } \\
\text { château, clou, comble, cour, } \\
\text { embrasure, étage, fenêtrage, } \\
\text { frise, herse, jambe, ligne, } \\
\text { maçon, mortier, niveau, } \\
\text { paroi, pavé, pivot, place, } \\
\text { plane, planche, plancher, } \\
\text { plomb, porte, poutre, saper, } \\
\text { sommier, tuile }\end{array}$ & $\begin{array}{l}8 \text { mots : chas, } \\
\text { embrasser, étalon, } \\
\text { frit, marqueter, } \\
\text { plant, plomber, } \\
\text { sole, }\end{array}$ & $\begin{array}{l}4 \text { mots : hie, } \\
\text { huis, mitoyen, } \\
\text { rez }\end{array}$ \\
\hline $\begin{array}{c}85 \\
(100 \%) \\
\end{array}$ & $\begin{array}{c}7 \\
\left(8,2^{\circ} \%\right) \\
\end{array}$ & $\begin{array}{c}35 \\
(41,1 \%) \\
\end{array}$ & $\begin{array}{c}8 \\
7,5 \% \\
\end{array}$ & $\begin{array}{c}4 \\
(5 \%) \\
\end{array}$ \\
\hline
\end{tabular}

Nous détectons ainsi un premier groupe composé par 7 mots du corpus sans marquage diatechnique, mais dont les acceptions font référence au domaine de l'architecture et du bâtiment. Néanmoins, il faut remarquer que le style définitoire de ces termes ne correspond pas à celui des termes qui portent un marquage diatechnique ni non plus au groupe de termes identifiés avec une seule acception liée au domaine de l'architecture et le bâtiment. De cette façon, les définitions de ce groupe ne sont ni concises ni univoques et elles sont accompagnées d'exemples. On peut apprécier ce type de définition dans l'entrée plinthe : 
PLINTHE. s. f. (Quelques-uns le font masculin.) Membre d'Architecture ayant la forme d'une petite table carrée, qui se nomme aussi Socle dans les bases, \& Tailloir dans les chapiteaux des colonnes. La plinthe de cette base n'a pas de proportion avec la plinthe du chapiteau. Il se dit aussi Des plates-bandes qui règnent dans les ouvrages de maçonnerie \& de menuiserie; \& alors il n'est que féminin. Cette plinthe est trop étroite, \& n'a pas assez de saillie.

Comme nous pouvons l'observer, toutes les acceptions de cette entrée font référence à l'architecture. Néanmoins, les définitions ne sont pas précises. Nous trouvons ainsi, par exemple, l'expression «Il se dit aussi », expression complémentaire nécessaire due au manque de précision. En ce qui concerne les exemples, on remarque que l'article propose plusieurs exemples d'utilisation : cette plinthe est trop étroite, \& n'a pas assez de saillie.

Même si ces termes appartiennent à un domaine spécialisé, ces entrées n'ont pas fait l'objet du même travail lexicographique que les précédentes. Nous constatons donc que l'Académie les a répertoriées sans suivre le style définitoire qu'elle utilise pour les termes considérés comme techniques. À notre avis, ces mots auraient mérité un travail plus approfondi et possiblement un marquage diatechnique.

Un autre groupe sans marquage diatechnique est formé de 35 mots. Ces entrées ont également plusieurs acceptions mais, à la différence du groupe précédent, au moins une n'est pas liée ni au domaine du bâtiment ni de l'architecture. Ces entrées comprennent également des exemples. Nous retrouvons ainsi :

ASTRAGALE $:$ s.m. Ornement d'Architecture fait ordinairement en forme de baguette, \& quelquefois taillé en petites boules, ou en grains de chapelet enfilés, \& qu'on emploie aux chapiteaux \& aux corniches. L'astragale se met ordinairement audessus d'un quart de rond. Un chapiteau orné d'astragales. Les Anatomistes appellent Astragale, Un des os du talon. Plante légumineuse. Sa racine est douce au goût. Quelques-uns par cette raison la nomment fausse Réglisse.

En effet, dans cette entrée nous pouvons remarquer comment la première acception fait une référence directe à l'architecture avec une information spécialisée, même si le dernier exemple qu'elle propose fait référence au domaine de l'anatomie. La deuxième acception, par contre, n'a aucun rapport avec le domaine technique de l'architecture ou du bâtiment. À notre avis, tenant compte du type de définition et du système de marques employés par l'Académie, cette entrée devrait contenir la marque " EN TERMES DE...».

De même, nous constatons un autre groupe formé par 8 mots du corpus qui figurent dans le D.A. sans qu'aucune de leurs acceptions ne soit liée aux domaines de l'architecture et du bâtiment. Par exemple : 
Le vocabulaire de l'architecture dans le Dictionnaire de l'Académie française (1762) : après les traces de Nicot

ÉTALON. s.m. Cheval entier, qui sert, qu'on emploie à couvrir des cavales. $\mathrm{Ce}$ cheval est bon à servir d'étalon. Il avoit tant d'étalons dans son haras. Bel étalon. Modèle, prototype de poids, de mesures, qui est réglé, autorité \& conservé par le Magistrat, \& sur lequel les mesures, les poids des Marchands doivent être ajustés, rectifiés, égalés. Étalon d'aune, de boisseau, de pot, de livre. L'étalon est marqué de la marque du Seigneur.

Comme nous pouvons le remarquer avec l'exemple ci-dessus, aucune des acceptions n'est liée directement aux domaines de l'architecture ni du bâtiment et aucune information contenue dans l'article ne fait référence à un domaine spécialisé. Cela démontrerait que ces mots ont perdu leur caractère spécialisé exclusif en devenant des mots du langage courant, même s'ils continuent à être aussi utilisés dans les domaines techniques.

Et pour finir, nous avons identifié un dernier groupe composé de 4 mots dont les définitions nous indiquent qu'ils sont obsolètes ou qu'ils ont été remplacés par d'autres termes. Nous trouvons ainsi par exemple :

HUIS. s. m. Porte. Ce mot est vieux, \& il n'a plus guère d'usage que dans ces phrases de Pratique, Tenir l'audience à huis clos, à huis ouverts.

Ou bien :

REZ. Préposition. Tout contre, joignant. Elle n'a plus d'usage que dans cette phrase, Rez pied, rez terre, qui signifie, Joignant la terre, à fleur de terre. On a abattu cette maison, cette place, ces fortifications rez pied, rez terre.

Comme nous pouvons l'apprécier dans ces exemples, les différentes acceptions de ces mots nous indiquent que, même si ces mots figurent dans le D.A., sont qualifiés comme des termes obsolètes, bien que leur usage soit encore en vigueur pour quelques locutions.

Afin de vérifier si ces mots étaient encore en usage à l'époque, nous avons consulté deux dictionnaires spécialisés, le Dictionnaire portatif des beaux-arts (1752) de Jacques Lacombe et le Dictionnaire d'architecture civile, militaire et moderne et de tous les arts et métiers qui en dépendent (1770), de Roland le Virloys.

Tableau 7. Présence de mots recueillis par le D.A. comme obsolètes dans deux dictionnaires spécialisés

\begin{tabular}{|l|c|c|c|c|}
\hline & Hie & Huis & Mitoyen & Rez \\
\hline $\begin{array}{l}\text { Dictionnaire portatif des beaux-arts } \\
\text { (1752) }\end{array}$ & $\varnothing$ & $\varnothing$ & $\varnothing$ & $\varnothing$ \\
\hline $\begin{array}{l}\text { Dictionnaire d'architecture civile, mili- } \\
\text { taire et moderne et de tous les arts et } \\
\text { métiers qui en dépendent (1770) }\end{array}$ & $\begin{array}{c}\text { Voyez MOU- } \\
\text { TON \& DEMO- } \\
\text { SISELLE }\end{array}$ & $\begin{array}{c}\text { Vieux terme } \\
\text { français, qui } \\
\text { signifie porte }\end{array}$ & $\varnothing$ & $\varnothing$ \\
\hline
\end{tabular}


Comme nous pouvons l'apprécier dans ce tableau, aucun de ces 4 mots ne figure dans le Dictionnaire portatif des beaux-arts (1752) et seulement Hie et Huis font partie de la nomenclature du Dictionnaire d'architecture civile, militaire et moderne et de tous les arts et métiers qui en dépendent (1770). Le mot Hie nous renvoie ainsi à des mots plus récents et le mot Huis est répertorié comme un mot désuet. Cette constations renforce l'aspect synchronique du travail lexicographique de l'Académie.

\section{7. Étude comparative des articles concernant les termes du corpus avec marquage technique appartenant aux domaines du bâtiment et de l'architecture en relation aux autres dictionnaires techniques}

Pour conclure notre analyse, nous allons étudier les différentes définitions des mots de notre corpus présents dans le D.A. qui possèdent le marquage diatechnique «TERME DE... » ou «EN TERMES DE... » et qui sont liés au domaine de l'architecture et du bâtiment, soit 5 termes en tout : Dosseret, Larmier (TERMES D'ARCHITECTURE), Moise (TERME DE CHARPENTERIE), Queue d'aronde (TERME DE MENUISERIE) et Assemblage (EN TERMES DE MENUISERIE). Nous allons ainsi comparer les définitions de l'Académie avec les définitions du D.N. et les deux dictionnaires spécialisés en architecture, le Dictionnaire portatif des beaux-arts (1752) de Jacques Lacombe, dorénavant DPBA, et le Dictionnaire d'architecture civile, militaire et moderne et de tous les arts et métiers qui en dépendent (1770), de Roland le Virloys, dorénavant DACMM.

Le but de cette analyse est d'étudier le degré de technicité et de spécialisation ainsi que la précision de ces définitions. De même, cette comparative nous permettra d'analyser de quelle manière les différentes acceptions de ces mots ont évolué ou non depuis Nicot, et de mettre en lumière le travail lexicographique des rédacteurs de ces différents dictionnaires. Le tableau suivant recueille les différentes définitions concernant l'entrée dosseret: 
Tableau 8. Comparaison des différentes définitions du mot dosseret

\begin{tabular}{|l|l|}
\hline $\begin{array}{l}\text { Thresor de la langue françoyse, } \\
\text { tant ancienne que moderne, de Jean } \\
\text { Nicot } \\
(\mathbf{1 6 0 6}) \text { - D.N. }\end{array}$ & $\begin{array}{l}\text { DOSSERASSE, au chap. 6. art. 11. des coustu. de. Par. } \\
\text { Il n'est loisible à un voisin mettre poultres dedans le mur } \\
\text { moitoyen sans y mettre jambes, parpaignes ou dosserasses, } \\
\text { cheynes et corbeaux suffisans de pierre de taille pour porter } \\
\text { lesdites poutres. }\end{array}$ \\
\hline $\begin{array}{l}\text { Dictionnaire portatif des beaux-arts } \\
\text { (1752) - DPBA }\end{array}$ & $\varnothing$ \\
\hline $\begin{array}{l}\text { Dictionnaire de l'Académie } \\
\text { (1762) - D.A. }\end{array}$ & $\begin{array}{l}\text { DOSSERET. s.m. } \\
\text { TERME D'ARCHITECTURE. Petit pilastre saillant. }\end{array}$ \\
\hline $\begin{array}{l}\text { Dictionnaire d'architecture civile, } \\
\text { militaire et moderne et de tous les } \\
\text { arts et métiers qui en dépendent } \\
(\mathbf{1 7 7 0 )} \text { - DACMM }\end{array}$ & $\begin{array}{l}\text { DOSSERET f. m. } \\
\text { Est un petit bout de mur en équerre, fur un autre, lequel sert } \\
\text { de jambage à une porte, ou une croisée, où à porter de fond } \\
\text { un arc doubleau }\end{array}$ \\
\hline
\end{tabular}

Pour le mot dosseret il faut signaler la grande différence entre les définitions des dictionnaires analysés. En ce qui concerne la définition du D.A., par rapport aux deux autres, elle est beaucoup plus précise. En effet, cette définition se compose d'une seule phrase avec laquelle les Académiciens parviennent à résumer et à délimiter le concept avec justesse. La définition du DACMM, en revanche, est plus longue et suit la ligne définitoire, explicative et descriptive, du D.N.

Voyons, maintenant, l'exemple de larmier : 
Tableau 9.Comparaison des différentes définitions du mot larmier

\begin{tabular}{|c|c|}
\hline $\begin{array}{l}\text { Thresor de la langue françoyse, tant } \\
\text { ancienne que moderne, de Jean Nicot } \\
\text { (1606) - D.N. }\end{array}$ & $\begin{array}{l}\text { LARMIER, m. acut. } \\
\text { Est la jettée de la tuyle ou autre chose issant du couvert } \\
\text { d'une maison outre l'aplomb de la muraille de dessous, } \\
\text { pour jetter le desgoust coulant de la couverture au loing } \\
\text { d'icelle muraille, et garder que ledit desgoust ne la cor- } \\
\text { rompe. } \\
\text { Et est un mot usité en maçonnerie, par imitation de ce } \\
\text { mot Latin Lacryma, que le François dit Larme, comme si } \\
\text { lon disoit Lacrymarium, par ce que les gouttes cheant des } \\
\text { tuyles semblent à des larmes decoulans des yeux, } \\
\text { Quia guttas stillicidij ceu lachrymas cadentes reiicit a pariete. } \\
\text { Le larmier d'une muraille, Proiectura, Supercilium. } \\
\text { Les larmiers d'une maison, } \\
\text { Coronae. Budaeus praecincturas etiam vocat, et praecinc- } \\
\text { tiones. } \\
\text { Larmier aussi entre mareschaux de chevaux est la vene } \\
\text { plus proche de l'oeil du cheval, ainsi appelée de ce qu'elle } \\
\text { joint l'oeil d'iceluy, } \\
\text { Vena ocularis. }\end{array}$ \\
\hline $\begin{array}{l}\text { Dictionnaire portatif des beaux-arts } \\
\text { (1752) - DPBA }\end{array}$ & $\begin{array}{l}\text { LARMIER. } \\
\text { TERME D'ARCHITECTURE. C'eft le plus fort membre } \\
\text { quarré d'une corniche dont le plafond eft fouvent creufé, } \\
\text { afin de faire égouter l'eau loin du mur en la faifant tomber } \\
\text { goûte à goûte comme des larmes. }\end{array}$ \\
\hline $\begin{array}{l}\text { Dictionnaire de l'Académie } \\
\text { (1762) - D.A. }\end{array}$ & $\begin{array}{l}\text { LARMIER. s.m. } \\
\text { Pièce de bois mise en saillie au bas d'un châssis, pour em- } \\
\text { pêcher que l'eau ne coule dans l'intérieur de la chambre. } \\
\text { TERME D'ARCHITECTURE, qui se dit d'une saillie qui } \\
\text { est hors de l'aplomb de la muraille, \& qui sert à empêcher } \\
\text { que l'eau ne découle le long du mur. } \\
\text { Dans une corniche, se dit aussi de la partie qui est le plus } \\
\text { en saillie. }\end{array}$ \\
\hline $\begin{array}{l}\text { Dictionnaire d'architecture civile, mili- } \\
\text { taire et moderne et de tous les arts et } \\
\text { métiers qui en dépendent } \\
\text { (1770) - DACMM }\end{array}$ & $\begin{array}{l}\text { LARMIER f. m. } \\
\text { Lat. Corona, Ail. Krans-leifle. } \\
\text { Est le plus fort membre quarré d'une corniche, dont le } \\
\text { plafond est ordinairement creusé en canal, pour faire égou- } \\
\text { ter l'eau, ou la faire tomber goutte à goutte, comme des } \\
\text { larmes, loin du mur qui est au-dessus. Le bord extérieur de } \\
\text { ce canal, se nomme mouchette, mais les ouvriers appellent } \\
\text { mouchette le larmier même. } \\
\text { On l'appelle aussi couronne goutière. }\end{array}$ \\
\hline
\end{tabular}

Lorsque nous analysons les définitions apportées par les deux dictionnaires spécialisés, nous constatons qu'elles présentent de nombreuses similitudes avec le D.N. 
Nous avons souligné, par exemple, l'expression larmes decoulans des yeux, métaphore utilisée par Nicot pour décrire la façon dont l'eau tombe et qui est reprise presque de manière identique dans le DPBA et dans le DACMM. Ainsi, on pourrait presque affirmer qu'une des sources de ces deux dictionnaires pour les termes de l'architecture et du bâtiment est le répertoire de Nicot. L'Académie, de sa part, n'utilise pas cette métaphore de Nicot et sa définition du mot LARMIER est plus précise, claire et concise.

Voyons, aussi, l'exemple de moise :

Tableau 10. Comparaison des différentes définitions du mot moise

\begin{tabular}{|c|c|}
\hline $\begin{array}{l}\text { Thresor de la langue françoyse, tant } \\
\text { ancienne que moderne, de Jean Nicot } \\
\text { (1606) - D.N. }\end{array}$ & $\begin{array}{l}\text { MOISE, f. pen. } \\
\text { En fait de charpenterie est une piece de bois de demie } \\
\text { espaisseur d'une poultre, si que les deux moises joinctes } \\
\text { ensemble rendent l'espaisseur d'une poultre, et est dite } \\
\text { Moise quasi la moitié d'une poultre quant à l'espaisseur } \\
\text { seulement, et les charpentiers usent de deux moises joinctes } \\
\text { ensemble en lieu de poultre, en montans ou en croix de S. } \\
\text { André (d'autant que la moise ainsi appliquée est en son } \\
\text { fort) et non en bois couché de long, } \\
\text { Semitrabs. }\end{array}$ \\
\hline $\begin{array}{l}\text { Dictionnaire portatif des beaux-arts } \\
\text { (1752) - DPBA }\end{array}$ & $\varnothing$ \\
\hline $\begin{array}{l}\text { Dictionnaire de l'Académie } \\
\text { (1762) - D.A. }\end{array}$ & $\begin{array}{l}\text { MOISE. s.f. } \\
\text { TERME DE CHARPENTERIE. Pièce de bois qui sert à } \\
\text { lier ensemble d'autres pièces, telles que les pieux d'un pont, } \\
\text { ou les pièces droites ou inclinées d'une grue, d'un engin. }\end{array}$ \\
\hline $\begin{array}{l}\text { Dictionnaire d'architecture civile, mili- } \\
\text { taire et moderne et de tous les arts et } \\
\text { métiers qui en dépendent } \\
\text { (1770) - DACMM }\end{array}$ & $\begin{array}{l}\text { MOISE, f. f. } \\
\text { Est toute pièce de bois jumelle qui sert à entretenir plu- } \\
\text { sieurs autres pièces d'un assemblage de charpente, soit } \\
\text { d'équerre, soie obliquement, \& qui à cet effet est entaillée } \\
\text { ou délassée pour les accoler. Chaque moise jumelle est } \\
\text { jointe par des tenons \& mortaises, \& chevillée. }\end{array}$ \\
\hline
\end{tabular}

En ce qui concerne le mot moise, on constate comment la définition qui figure dans le D.N. est longue et explicative, car elle montre différents exemples d'utilisations et d'applications de ce mot. Quant à la définition du Dictionnaire de l'Académie on observe qu'elle n'est pas si juste et univoque que dans le cas du terme larmier. Cependant, elle est plus claire et concise que celle du Nicot et il faut signaler qu'elle n'apporte pas d'exemples d'utilisation. En ce qui concerne la définition du DACMM, elle est plus longue et illustrative et, bien qu'il s'agisse d'un dictionnaire spécialisé, l'article suit le même modèle explicatif du Nicot.

Voyons à continuation le mot queue d'aronde : 
Tableau 11. Comparaison des différentes définitions du mot queue d'aronde

\begin{tabular}{|l|l|}
\hline $\begin{array}{l}\text { Thresor de la langue françoyse, tant } \\
\text { ancienne que moderne, de Jean Nicot } \\
\text { (1606) - D.N. }\end{array}$ & $\begin{array}{l}\text { QUEUE D'ARONDE OU D'ARONDELLE } \\
\text { En cas de serrurerie ou charpenterie est une piece de fer } \\
\text { ou de bois large et espanie és deux extremitez, estroicte au } \\
\text { milieu, servant à joindre par enchassure deux pieces de } \\
\text { bois ensemble, ou bien le bout d'une piece de bois taillé } \\
\text { en queue d'arondelle pour enchasser dans le bout d'une } \\
\text { autre piece qu'on veut joindre et adapter à celle-là, } \\
\text { Le mot est ainsi dit, parce que laditte piece à enchasser } \\
\text { et joindre represente la forme d'une queue d'arondelle } \\
\text { double ou simple, } \\
\text { Securicla, Subscus. }\end{array}$ \\
\hline $\begin{array}{l}\text { Dictionnaire portatif des beaux-arts } \\
\text { (1752) - DPBA }\end{array}$ & $\begin{array}{l}\varnothing \\
\text { Dictionnaire de l'Académie } \\
\text { (1762) - D.A. }\end{array}$ \\
$\begin{array}{l}\text { QUEUE D'ARONDE. } \\
\text { TERME DE MENUISERIE, qui se dit d'un certain } \\
\text { tenon, d'une certaine pièce de liaison taillée en queue } \\
\text { d'hirondelle. }\end{array}$ \\
\hline $\begin{array}{l}\text { Dictionnaire d'architecture civile, mili- } \\
\text { taire moderne et de tous les arts et } \\
\text { (1770) - DACMM }\end{array}$ & $\begin{array}{l}\text { QUEUE D'HIRONDE } \\
\text { Est la manière de tailler l'extrémité d'une pierre d'une } \\
\text { pièce de bois ou de fer pour l'àssembler avec une autre, en } \\
\text { faisant l'assemblage plus large à l'extrémité qu'au collet. }\end{array}$ \\
\hline
\end{tabular}

La définition du Nicot pour l'entrée queue d'aronde est, comme dans les cas précédents, plus explicative et illustrative, voir encyclopédique. Les définitions qui figurent dans le D.A. et le DACMM, sont plus courtes que dans le Thresor. Néanmoins on peut apprécier que la définition qui se trouve dans le D.A. est, dans ce cas, moins précise. En effet, l'utilisation du déterminant « certain/e » (certain tenon ... certaine pièce ...) dénote un manque de précision. De même, l'article décrit la forme particulière d'un objet sans spécifier son utilisation ou champ d'application. La définition concise, claire et univoque est par conséquent un peu moins de rigueur dans cet article. De sa part, dans le Dictionnaire d'architecture civile, militaire et moderne et de tous les arts et métiers qui en dépendent, on peut apprécier comment l'article décrit un procédé (une manière de tailler...) d'une manière également peu précise, sans spécifier non plus le champ d'application ou l'utilisation.

Étudions le cas du mot assemblage: 
Le vocabulaire de l'architecture dans le Dictionnaire de l'Académie française (1762) :

après les traces de Nicot

Tableau 12. Comparaison des différentes définitions du mot assemblage

\begin{tabular}{|l|l|}
\hline $\begin{array}{l}\text { Thresor de la langue françoyse, tant } \\
\text { ancienne que moderne, de Jean Nicot } \\
\text { (1606) - D.N. }\end{array}$ & $\begin{array}{l}\text { ASSEMBLAGE m. penac. } \\
\text { Est l'oeuvre de assembler les pieces en un corps. } \\
\text { Selon ce on dit, Il besongne à l'assemblage de la chaire. } \\
\text { Compagini partium cathedrae incumbit. } \\
\text { Il se prend aussi pour iceluy ouvrage fait, ainsi on dit, } \\
\text { l'assemblage en est beau. } \\
\text { Partium compago apta ac concinna est. } \\
\text { Coagmentum, Compago, Commissio, Iunctura, Coagmenta- } \\
\text { tio. }\end{array}$ \\
\hline $\begin{array}{l}\text { Dictionnaire portatif des beaux-arts } \\
\text { (1752) - DPBA }\end{array}$ & $\varnothing$ \\
\hline $\begin{array}{l}\text { Dictionnaire de l'Académie } \\
\text { (1762) - D.A. }\end{array}$ & $\begin{array}{l}\text { ASSEMBLAGE. S. m. } \\
\text { Amas \& union de plusieurs choses qu'on joint ensemble. } \\
\text { Un bateau se fait de l'assemblage de plusieurs pièces de } \\
\text { bois. } \\
\text { Assemblage, en termes de Menuiserie, se dit De la manière } \\
\text { d'assembler le bois de menuiserie, \& des pièces principales } \\
\text { qui servent à cet effet. L'assemblage de cette porte ne vaut } \\
\text { rien. Bois d'assemblage. Porte d'assemblage. } \\
\text { Il se dit aussi des choses morales. Son caractère est un } \\
\text { assemblage de bonnes \& de mauvaises qualités. }\end{array}$ \\
\hline $\begin{array}{l}\text { Dictionnaire d'architecture civile, } \\
\text { militaire et moderne et de tous les arts } \\
\text { et métiers qui en dépendent } \\
\text { (1770) - DACMM }\end{array}$ & \begin{tabular}{l}
$\varnothing$ \\
\hline
\end{tabular} \\
\hline
\end{tabular}

Pour le mot assemblage signalons, tout d'abord, qu'aucun des deux dictionnaires spécialisés ne recueille ce mot, ce qui indiquerait que ces deux dictionnaires ne le considèrent pas comme un mot technique propre au vocabulaire de l'architecture. En ce qui concerne la définition du Nicot, on voit comme elle nous renvoie au mot assembler en utilisant l'expression «l'œuvre de assembler...(sic.) ». Il faut remarquer, également, que dans cette occasion l'article est relativement court par rapport aux entrées précédentes. Quant à l'Académie, elle recueille ce mot dans son dictionnaire avec le marquage « EN TERMES DE... ». La définition du terme, malgré son marquage diatechnique, est moins précise que dans les cas précédents. De même, il faut souligner que la définition contient des exemples, L'assemblage de cette porte ne vaut rien, et fait allusion également au sens imagé du mot, Il se dit aussi des choses morales. Son caractère est un assemblage de bonnes \& de mauvaises qualités. Nous pouvons conclure que, d'après le style définitoire de l'Académie, ce mot est en train de se banaliser et l'Académie a utilisé la marque «EN TERMES DE... » pour remarquer que le terme se trouve en transition entre le langage spécialisé et la langue courante.

Pour conclure, analysons l'entrée assembler : 
Tableau 13. Comparaison des différentes définitions du mot assembler

\begin{tabular}{|c|c|}
\hline $\begin{array}{l}\text { Thresor de la langue françoyse, tant } \\
\text { ancienne que moderne, de Jean Nicot } \\
\text { (1606) - D.N. }\end{array}$ & $\begin{array}{l}\text { ASSEMBLER Qui s'assemble facilement, Congregabilis. } \\
\text { Maison où il s'assemble grand peuple, Frequentissima } \\
\text { aedes. } \\
\text { Assemblé, m. acut. Collectus. Coadunatus, Coactus, } \\
\text { Coagmentatus. B. Compositus. } \\
\text { Tout assemblé, Cunctus. } \\
\text { Assemblé et joinct, Conciliatus. } \\
\text { Qui est fait et assemblé de plusieurs choses qui sont } \\
\text { comme aggluées ensemble, Concretum, Compactum, } \\
\text { Conglutinatum, Coagmentatum. } \\
\text { Gens assemblez et de toutes pieces, Collectanei homines. B. } \\
\text { Qui vndiquaque conuenerunt. }\end{array}$ \\
\hline $\begin{array}{l}\text { Dictionnaire portatif des beaux-arts } \\
\text { (1752) - DPBA }\end{array}$ & $\varnothing$ \\
\hline $\begin{array}{l}\text { Dictionnaire de l'Académie } \\
\text { (1762) - D.A. }\end{array}$ & $\begin{array}{l}\text { ASSEMBLER. v.a. Mettre ensemble. Assembler des } \\
\text { troupes. Assembler les États. Assembler des matériaux } \\
\text { pour bâtir. Assembler des papiers, des livres. Assembler les } \\
\text { feuilles d'un livre pour le relier. ASSEMBLER, se dit aussi } \\
\text { en termes de Menuiserie \& de Charpenterie, pour dire, } \\
\text { joindre, emboîter, enchasser plusieurs pièces de bois, en- } \\
\text { sorte qu'elles ne fassent qu'un corps. Assembler des pièces } \\
\text { de charpente, de menuiserie, \&c. On dit proverbialement, } \\
\text { qu'un homme a bientôt assemblé son conseil, pour dire, qu'il } \\
\text { prend brusquement ses résolutions sans consulter per- } \\
\text { sonne. ASSEMBLER, est aussi réciproque. Le Parlement } \\
\text { s'assembla. Le peuple s'assemble. Les créanciers de cette } \\
\text { direction s'assemblent deux fois la semaine. ASSEMBLE,, } \\
\text { ÉE. participe. }\end{array}$ \\
\hline $\begin{array}{l}\text { Dictionnaire d'architecture civile, mili- } \\
\text { taire et moderne et de tous les arts et } \\
\text { métiers qui en dépendent } \\
\text { (1770) - DACMM }\end{array}$ & $\begin{array}{l}\text { ASSEMBLER v. a. Joindre ensemble les différentes } \\
\text { pièces de bois de charpente, préparées \& taillées pour la } \\
\text { conftruction d'un pan de bois, d'un comble, \&c. \& en } \\
\text { menuiferie, pour former une porte, un chaffis de croifée, } \\
\text { un lambris, \&c. }\end{array}$ \\
\hline
\end{tabular}

L'entrée assembler n'est pas présent dans le DPBA, tandis que chez Nicot, nous trouvons plus une énumération d'exemples qu'une définition. L'Académie, de sa part, fait référence à un usage spécialisé dans la deuxième acception en utilisant la marque «EN TERMES DE... ». Il s'agit d'une définition précise que présente un exemple d'utilisation. De même, le DACMM définit le terme avec concision et précision sans apporter des exemples d'utilisation, bien qu'il explique les différents usages en fonction du domaine auquel on se rapporte. Des trois définitions, celle qui est la plus claire et qu'en même temps propose une information riche et pertinente est celle du DACMM.

L'analyse de ces six entrées dans les quatre dictionnaires étudiés, démontre que les définitions du répertoire institutionnel sont celles qui présentent un degré de technicité 
majeur : des définitions univoques et concises sans faire appel au langage figuré, tandis que les dictionnaires spécialisés se rapprochent plus du modèle définitoire du Dictionnaire de Nicot, plus descriptif et moins précis.

\section{Conclusions}

Tout au long de cet article, nous avons suivi les traces de Nicot dans le Dictionnaire de l'Académie (1762) et, pour cela, nous avons étudié la liste de 94 termes relevés par Wooldrige (1985) recueillant l'essentiel du vocabulaire du bâtiment et de l'architecture du Dictionnaire de Nicot (1606).

Lors de notre étude, nous avons constaté que 9 mots des 94 termes qui constituent le corpus ne sont pas présent dans le D.A. Afin de vérifier si ces termes étaient encore en usage à l'époque, nous avons consulté deux dictionnaires d'architecture et du bâtiment contemporains au D.A., le Dictionnaire portatif des beaux-arts (1752) et le Dictionnaire d'architecture civile, militaire et moderne et de tous les arts et métiers qui en dépendent (1770). Nous avons donc vérifié que ces 9 termes ne sont pas non plus présents dans ces dictionnaires spécialisés, ce qui démontre que le travail de l'Académie répond aux standards de vitalité synchronique des mots répertoriés.

De même, pour notre analyse, nous avons tenu compte des possibles modifications orthographiques des 85 termes de notre corpus répertoriés dans le D.A. Nous avons ainsi attesté qu'effectivement, 33 de ces 85 termes ont subi des modifications orthographiques et sont recensés dans le D.A. avec une graphie différente, ce qui est en accord avec les modifications orthographiques et normatives qui ont eu lieu tout au long des $\mathrm{XVII}^{\mathrm{e}}$ et XVIII ${ }^{\mathrm{e}}$ siècles.

En ce qui concerne l'étude des articles et le système de marquage du D.A., notre analyse démontre que l'Académie en utilise un, malgré l'absence de toute référence dans la préface de l'ouvrage. En effet, l'Académie n'explique pas la structuration de son système de marquage, mais elle répertorie certains mots spécialisés avec le marquage « TERME DE... » ou « EN TERMES DE... ». D'après notre étude, les mots recensés avec la marque "TERME DE... » contiennent une définition univoque, sans exemples ni sens figuré, ce qui indique qu'il s'agit de termes spécialisés. Nous trouvons ainsi 4 mots parmi ces 85 termes recueillis dans le D.A. avec un marquage diatechnique liée au domaine du bâtiment et de l'architecture. En ce qui concerne la marque «EN TERMES DE... », on peut affirmer que l'Académie ne répertorie que 2 termes liés au domaine de la construction et l'architecture. Le reste de termes répertoriés sous ce marquage sont identifiés comme des termes propres à d'autres domaines spécialisés. L'Académie utilise ce marquage pour signaler des termes dont une des acceptions est encore spécialisée, c'est-à-dire, les autres acceptions qui constituent l'article sont des acceptions qui montrent un usage non spécialisé du terme. On peut donc affirmer que les termes qui portent cette marque sont en voie de se généraliser.

Il faut signaler, cependant, que ce système de marquage n'est pas complètement stable, car, comme nous l'avons souligné, il existe certaines entrées du répertoire dont la définition correspond au modèle définitoire des termes portant la marque «TERMES 
DE... ", mais qui ne possèdent pas de marquage diatechnique. De cette manière, nous avons constaté la présence de 21 mots qui ne portent pas cette marque, mais dont leurs définitions suivent ce modèle.

De plus, dans notre travail, nous distinguons un autre groupe de 7 mots qui auraient probablement mérité un autre traitement lexicographique et le marquage diatechnique «TERME DE... ». En effet, même si ces entrées comprennent plusieurs acceptions, certes moins précises, toutes nous renvoient au domaine spécialisé de l'architecture ou du bâtiment. De même, nous avons remarqué que 35 autres mots sont en voie de se banaliser et qu'ils auraient certainement dû être répertoriés avec le marquage " EN TERMES DE... » Comme nous l'avons montré, ces termes contiennent plusieurs acceptions dont au moins une n'est pas liée aux domaines du bâtiment et de l'architecture.

Il faut remarquer aussi le transfert de vocabulaire spécialisé d'un domaine technique à un autre. Ainsi, certains de ces mots ont été identifiés comme appartenant à des domaines techniques différents de celui de l'architecture : cuisine, anatomie, fauconnerie, entre autres.

Finalement, grâce à la comparaison des 6 articles qui figurent dans le D.A. marqués diatechniquement comme propres au domaine de l'architecture et du bâtiment avec les différents dictionnaires spécialisés contemporains au D.A, ainsi qu'avec les définitions du Dictionnaire de Nicot (1606), nous avons réussi à mettre en évidence le travail scientifique et lexicographique réalisé par les Académiciens. En effet, l'étude des définitions des termes analysés démontre que les Immortels, en général, ont abordé les définitions de ces mots en suivant un schéma définitoire innovateur dans cette époque : des définitions précises, univoques et sans exemples, ce qui n'était pas le cas d'autres dictionnaires techniques d'alors, qui suivent souvent le style définitoire de Nicot.

En raison de nos résultats, nous pouvons donc affirmer que la quatrième édition du répertoire institutionnel (1762) marque un point d'inflexion dans la lexicographie institutionnelle. Effectivement, l'Académie, soucieuse d'offrir au public un dictionnaire qui reflète la langue en usage répertorie dans son dictionnaire un grand nombre de mots techniques et scientifiques. De même, elle utilise un style définitoire innovant et un système de marquage, pas encore complètement systématisée, mais qui met en évidence la scientificité du travail lexicographique de l'Institution et qui devrait se développer au fil des éditions suivantes.

\section{BIBLIOGRAPHIE}

\section{Sources primaires}

ACADÉMIE FRANÇAISE. (1762) : Dictionnaire de L'Académie française, 4ème Edition. Paris : Vve B. Brunet.

LACOMBE, J. (1752) : Dictionnaire portatif des beaux-arts, ou Abrégé de ce qui concerne l'architecture, la sculpture, la peinture, la gravure, la poésie et la musique... Paris : Estienne et fils et J.-T. Hérissant.

ROLAND LE VIRLOYS, C.F. (1770) : Dictionnaire d'architecture civile, militaire et moderne et de tous les arts et métiers qui en dépendent, Paris : Libraires associés. 
Le vocabulaire de l'architecture dans le Dictionnaire de l'Académie française (1762) : après les traces de Nicot

NICOT, J. (1606) : Thresor de la langue francoyse tant ancienne que moderne : auquel... sont les mots propres de marine, vénerie et faulconnerie... Paris : David Douceur, libraire juré, ruë Saint Jaques, à l'enseigne du Mercure arresté. M. DC. VI

RICHELET, P. (1680) : Dictionnaire françois : contenant les mots et les choses, plusieurs nouvelles remarques sur la langue françoise, ses expressions propres, figurées et burlesques, la prononciation des mots les plus difficiles, le genre des noms, le régime des verbes... Genève, Suisse. J.-H. Widerhold.

\section{Sources secondaires}

BOULANGER, J.C.; L'HOME, M.C. (1991) : «Les technolectes dans la pratique dictionnairique générale. Quelques fragments d'une culture", Meta : Journal des traducteurs. 36(1): 23.

CORDERO MONGE, S. (2009) : "Algunas consideraciones sobre el lenguaje común y el lenguaje técnico”, Revista Káñina, XXXIII: 75-80.

COURBIERES, C.; FRAYSSE, P. (2009) : «Langages de l'architecture / architecture des langages : construction du sens dans le vocabulaire architectural», in : Intelligence collective et organisation des connaissances, Actes du 7ème Colloque du chapitre français de l'ISKO. Lyon: Université Jean Moulin Lyon 3 et ENSSIB: 239-248. [https://hal.archives-ouvertes.fr/hal-01234371]

ELUERD, R., (1999) : «Les vocabulaires scientifiques et techniques : Présence, emplois et réception», L'information grammaticale, 82(1): 62-71.

FARID, G., (2012) : «La « nouvelle orthographe », 21 ans plus tard». SHS Web of Conferences, 1: 2055-2069.

GIOVANI, M., (2006) : Les réformes de l'orthographe française. Une approche historique, contrastive et prospective. Université de Neuchâtel [online].

JOSSELIN-LERAY, A. (2005) : Place et rôle des terminologies dans les dictionnaires généraux unilingues et bilingues. Étude d'un domaine de spécialité : volcanologie. [online]. [http://theses.univ-lyon2.fr/documents/lyon2/2005/ josselin_a\#p=0\&a=top]

PHAL, A. (1969) : «La recherche au CRÉDIF : la part du lexique commun dans les vocabulaires scientifiques et techniques», Langue française, 2(1): 73-81.

QUEMADA, B. (1985) : «L'Académie française et ses dictionnaires : remarques sur la lexicographie institutionnelle française «, in The Fairest Flower. The Emergence of Linguistic National Consciousness in Renaissance Europe, International Conference of The Center for Medieval and Renaissance Studies, University of California, Los Angeles, 12-13 December 1983, Firenze: Firenze Presso l'Accademia: 71-84.

RIDAO RODRIGO, S.; RODRÍGUEZ MUÑOZ F.J. (2016) : “Evolución léxico-terminológica de las enfermedades raras: revisión de los diccionarios de la real academia española”. Alfa: Revista de Linguística, 60(1): 95-118.

RODRÍGUEZ ADRADOS, F. (1997) : "Los orígenes del lenguaje científico", Revista Espanola de Linguistica, (27): 299-317. 
ROFFIDAL, É. (2012) : «Architecture et théorie au XVIIIe siècle en France. La question de l'aménagement des choeurs d'églises». In : L.L. Sabine Frommel, ed. La place du choeur. Architecture et liturgie en Occident du Moyen âge aux Temps modernes. Paris, France: Picard. pp.227-235. [https://hal.archives-ouvertes.fr/hal-01557972]

ROULEAU, M. (2003) : «La terminologie médicale et ses problèmes», Panace@, $\operatorname{IV}\left(n^{\circ} 12\right): 10$.

SALLENAVE, D. (2016) : «L'orthographe : histoire d'une longue querelle (3)»| Page web de l'Académie française [online]. [http://www.academie-francaise.fr/lorthographe-histoire-dune-longue-querelle-3]

TERRAL, F. (2004) : «L'empreinte culturelle des termes juridiques», Meta : Journal des traducteurs, 49(4): 876-890.

TETET, C. (1994) : «La terminologie de l'alpinisme dans les dictionnaires», Meta : Journal des traducteurs, 39(4), pp.651-661.

TRUJILLO-GONZALEZ, V.C. (2019) : «Le discours préfaciel au XVIIIe siècle à travers le Dictionnaire de l'Académie (1718) et le Dictionnaire de Trévoux (1721). Essai de classification». Revue Romane. Langue et littérature. International Journal of Romance Languages and Literatures. [https://www.jbe-platform.com/content/journals/10.1075/rro.17014.tru]

TURCAN, I. ; WOOLDRIDGE, T.R., (2002) : «Le vocabulaire de la marine dans les dictionnaires anciens: éléments pour une base de donées cumulative». In: JADT 2002 : Gèmes journées internationales d'analyse statistique des données textuelles : 6th international conference on textual data statistical analysis : Saint-Malo, 1315 mars 2002 : Saint-Malo, march 13-15, 2002. JADT 2002 : 6es Journées internationales d'Analyse de Données Textuelles. Rennes, France: INRIA: 735-744.

WIONNET, W. (1998) : «Le discours sur l'Académie : entre rejet et convoitise», Actes du colloque international 17, 18 et 19 novembre 1994. ed. Lexica mots et dictionnaires 2. Paris Genève : Bernard Quemada (ed.) avec la collaboration de Jean Pruvost Honoré Champion.

WOOLDRIDGE, T.R. (1985) : «Le vocabulaire du bâtiment chez Nicot : quelques datations», Revue de linguistique romane, 49 (193-194): 327-358.

\section{PROFIL ACADÉMIQUE ET PROFESSIONNEL}

Zaida Bartolomé Díaz es Ingeniera Técnica de Obras Públicas por la ULPGC y posee el Grado en Ingeniería Civil por la UCAM. Además, ha cursado la licenciatura en Traducción e Interpretación en la ULPGC.

En estos momentos es estudiante de doctorado del programa Doctorado en Estudios Lingüísticos y Literarios en sus Contextos Socioculturales (DELLCOS) de la Universidad de Las Palmas de GC (España). Tras varios años en el sector de la ingeniería, se dedica actualmente en exclusiva a la traducción y la interpretación, principalmente en temáticas técnicas y científicas. 
Desde septiembre 2019, y hasta junio 2020, se encuentra realizando una estancia de investigación científica en el laboratorio Praxiling de la Université Paul Valéry de Montpellier.

Verónica C. Trujillo-González es doctora en filología francesa por la Universidad de Las Palmas de Gran Canaria, donde imparte docencia y es investigadora de la división Variación y Cambio Lingüístico del Instituto de Análisis y Aplicaciones Textuales (IATEXT). Sus principales líneas de investigación se centran en el estudio de obras lexicográficas, tanto desde una perspectiva sincrónica como diacrónica, con un especial interés por los repertorios multilingües del francés con la lengua china.

Fecha de recepción: 30/04/2020

Fecha de aceptación: 09/06/2020 